\title{
Der Regenwurm Dendrobaena illyrica (Cognetti, 1906) an der nördlichen und nordwestlichen Grenze seiner Verbreitung (Oligochaeta: Lumbricidae)
}

\author{
N. HÖSER \\ Norbert Höser, Am Park 1, D-04603 Windischleuba, Germany \\ E-Mail:norbert.hoeser@arcor.de
}

\begin{abstract}
The earthworm Dendrobaena illyrica (Cognetti, 1906) on the northern and northwestern boundary of its distribution (Oligochaeta: Lumbricidae). The northern and northwestern boundary of the range of Dendrobaena illyrica (Cognetti, 1906), which runs through the eastern part of the edge of the Central European Uplands, is here more precisely determined on the basis of new localities. The species has been found in areas of the Böhmerwald/Bayerischer Wald, Oberpfälzer Wald, Fichtelgebirge, Vogtland, Erzgebirge and Elbsandsteingebirge. On the northern slope of the Erzgebirge, the range boundary corresponds to the 'flint line' marking the margin of the first Elsterian Cold Period glaciation. This earthworm species prefers forest soils formed on acidic magmatites and metamorphites as source rocks. We found both fully developed individuals of the species which reproduce sexually, and externally not fully developed adult individuals which we classed as the parthenogenetic form of the species. The distribution of both forms shows that parthenogenesis appears to occur geographically in $D$. illyrica, i.e. the sexual form predominates in optimal habitats near the core of the range, while the parthenogenetic form occurs in less favourable habitats and at the edges of the range.
\end{abstract}

Keywords. Earthworms, Dendrobaena illyrica, Germany, new records, boundary of distribution, parthenogenesis.

\section{EINLEITUNG}

$\mathrm{B}$ ei den gut und gleichmäßig durchforschten Arten von Vertebraten, Insekten und höheren Blütenpflanzen sind fundierte Grenzen ihrer Areale ermittelt worden (de Lattin 1967, Müller 1977). Weniger günstig ist die Situation bei einigen Regenwurmarten, bei denen es noch zahlreicher einwandfreier faunistischer Unterlagen bedarf, um anhand von Fundpunkten Arealgrenzen feststellen zu können. Zu diesen Arten gehört Dendrobaena illyrica (Cognetti, 1906), von der angenommen wird (Lehmitz et al. 2016), sie sei in Deutschland extrem selten. Bisher sind in der faunistischen Literatur deutsche Funde der Art aus dem Bayerischen Wald/Hohen Böhmerwald (Michaelsen 1907), dem Oberpfälzer Wald (Makeschin 1983, 1987, Makeschin \& Beck 1986), dem westlichen Erzgebirge (Kollmannsperger 1936) und dem Osterzgebirge (Bösener 1964, 1965, La France 2002) bekannt. Zahlreiche Funde gibt es im angrenzenden tschechischen
Gebiet (z.B. Pižl 2001, 2002 a, b, Švarc \& Kula 2011, Kula \& Švarc 2012), wo D. illyrica in den Bergmischwäldern des Erzgebirges und Böhmerwaldes eine sub- bis eudominante Regenwurmart ist. Sie entspricht dem illyrischen Verbreitungstyp und wurde bisher in Deutschland, Tschechien, Österreich, Kroatien, Bosnien-Herzegowina, Montenegro, Serbien und Mazedonien nachgewiesen (Csuzdi et al. 2011, Szederjesi 2017; Abb. 1). Im vorliegenden Beitrag werden neue deutsche Funde und einige Beobachtungen zur Populationsstruktur der Art mitgeteilt.

\section{MATERIAL UND METHODEN}

\section{Untersuchungsgebiet}

Wir untersuchten Waldstandorte westlich der Elbe, so im Elbsandsteingebirge, Erzgebirgsvorland, Erzgebirge, Vogtland, Thüringer Schiefergebirge, Frankenwald, Fichtelgebirge, Oberpfälzer Wald und Bayerischen Wald, die sämtlich 


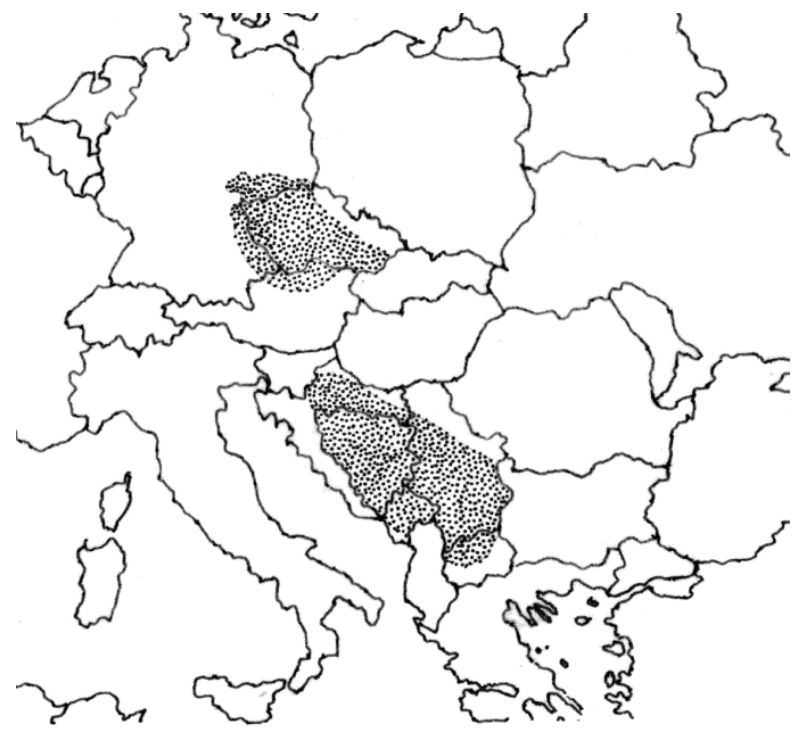

Abbildung 1. Die Verbreitung von Dendrobaena illyrica (Cognetti, 1906).

in Höhenlagen von mehr als $230 \mathrm{~m}$ ü. NN, meist höher als $400 \mathrm{~m}$ ü. NN im herzynisch-sudetischen Bergmischwaldgebiet (Freitag 1962) liegen. Die geprüften Bodenstandorte befinden sich in der natürlichen Zone der montanen und kollin-submontanen Buchenmischwälder (Firbas 1949, Walter \& Straka 1970) und im angrenzenden Randbereich der Eichen-Buchenwaldzone (Schmidt et al. 2002). Sporadisch untersucht wurden auch Standorte in den Nachbargebieten dieser Zone. Das betrachtete Untersuchungsgebiet, das ein Teil der östlichen mitteleuropäischen Mittelgebirgsschwelle ist, umfasst $c a .50000 \mathrm{~km}^{2}$.

\section{Methodik}

Hauptsächlich wurde die nordwestliche und nördliche Grenze des Areals von D. illyrica gesucht. Ausgehend von den bisher in der Literatur genannten Fundorten prüften wir deshalb vorwiegend in radialer Richtung weitere Bodenstandorte, um so an die Grenze des Areals der Art zu gelangen. Auf diese Weise wurden entlang der nördlichen Verbreitungsgrenze Standorte im Abstand von $c a$. 2 bis $10 \mathrm{~km}$ untersucht, an denen die Art vorkam oder fehlte. Selbstverständlich ist daher die Anzahl der Fundpunkte mitbestimmt vom Aufwand, diese Grenze zu finden. Um $D$. illyrica an einem Standort nachzuweisen, wurden
$0,25 \mathrm{~m}^{2}$ große Flächen genau geprüft, die überwiegend in der Regentraufe von Bäumen, an Hangfüßen oder in flachen Dellen mit etwas erhöhter Bodenfeuchte lagen. Zumeist waren es einzelne derartige Untersuchungsflächen, in einigen Fällen wurden zwei bis drei Flächen entlang eines Transekts untersucht. Bis in eine Tiefe von $0,3 \mathrm{~m}$ wurden alle Regenwürmer ausgegraben und in zweimaliger Durchsicht des Bodens von Hand ausgelesen. Die beim Ausgraben hergestellte Schürfgrube diente der Bodenansprache. Merkmale des Bodenprofils, der Geländemorphologie und der Vegetation des untersuchten Bodenstandorts wurden vor Ort protokolliert. Die Belege der gefundenen Regenwürmer befinden sich in der Sammlung des Autors.

\section{ERGEBNISSE}

\section{Das Verbreitungsbild der Art anhand der Verteilung der Fundpunkte}

Der Autor sammelte an 126 Fundpunkten 380 Individuen von $D$. illyrica. Auf den $0,25 \mathrm{~m}^{2}$ großen Bodenflächen dieser Fundpunkte konnten überwiegend einzelne, aber maximal 15 Individuen dieser Art festgestellt werden. D. illyrica wurde in Wäldern des östlichen Teils der Mitteleuropäischen Mittelgebirgsschwelle gefunden, nämlich am Südrand des mitteldeutschen Mulde-Lösshügellandes, im Elbsandsteingebirge, Erzgebirge, Erzgebirgsbecken, Vogtland einschließlich Ostthüringer Schiefergebirge und Elstergebirge, im Fichtelgebirge, Oberpfälzer Wald und Böhmerwald/Bayerischen Wald. Ungefähr auf einem Drittel aller in diesem Gebiet nach genannter Methode untersuchten Bodenstandorte kamen Nachweise der Art zustande. Dieses Ergebnis und die geographische Verteilung der von uns ermittelten Fundpunkte zeigen, dass im untersuchten Teil des Art-Areals sehr große, arealgliedernde Verbreitungslücken anscheinend nicht bestehen (Abb. 2). Somit ist davon auszugehen, dass D. illyrica in einem kontinuierlichen Areal vorkommt. Entlang der nördlichen Arealgrenze wurde eine Reihe von Fundpunkten ermittelt, die sich in kleineren und fleckenartig verteilten Waldhabitaten befinden. 


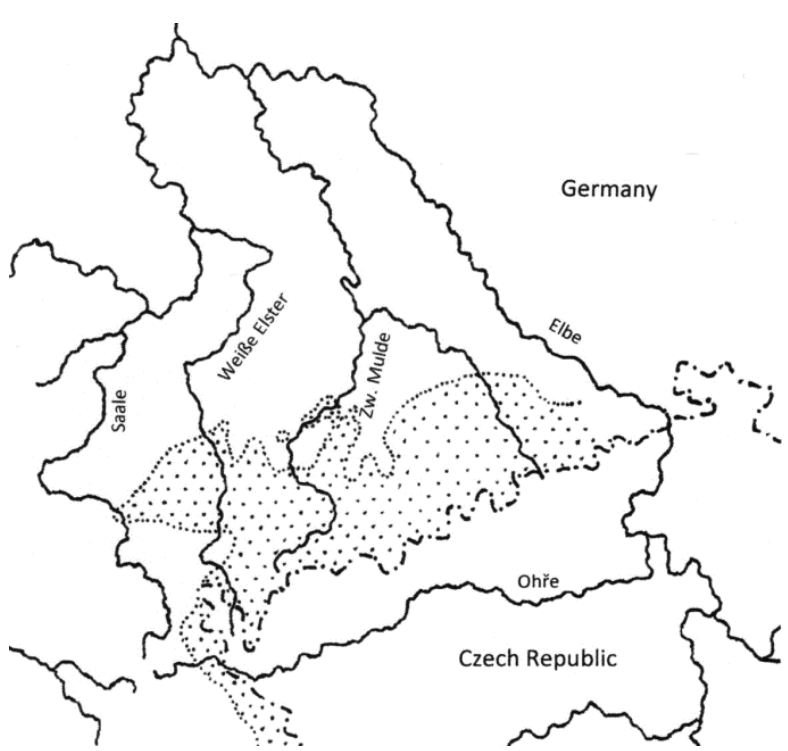

Abbildung 2. Der nördliche und nordwestliche Rand des Areals von Dendrobaena illyrica in Deutschland.

Keine Nachweise ergaben sich im Thüringer Schiefergebirge, im Frankenwald und auf der Münchberger Hochfläche, die nach gegenwärtiger Kenntnis vor dem Westrand des Areals liegen.

\section{Die nördliche und nordwestliche Verbreitungsgrenze}

An der Nordabdachung des Erzgebirges verläuft die anhand unserer Funde ermittelte Nordgrenze der Verbreitung von D. illyrica auf ca. $120 \mathrm{~km}$ Strecke zwischen Weißer Elster und Elbe annähernd deckungsgleich mit der Feuersteinlinie, die in Pietzsch (1962), Wagenbreth (1978), Eissmann (1994), Wolf \& Alexowsky (2008) und Ehlers (2011) annähernd gleich dargestellt ist. Diese Linie markiert den Rand der maximalen Elster-1-Kaltzeit-Vergletscherung, an der Zwickauer Mulde den Elster-2-Eisvorstoß. Mehrere nordwärts vorgeschobene Fundpunkte der Art entsprechen den Vorsprüngen der Feuersteinlinie, so im Hofholz bei Endschütz (4374), Trünziger Wald bei Wolframsdorf (4275), Jahnshorn bei Niederfrohna (4121), Viehwegbusch bei Bockendorf (4400) und Tharandter Wald bei Grund (4347). Diese Ergebnisse sprechen für eine Beziehung von $D$. illyrica zur Verwitterungsdecke, die sich im gletscherfreien Periglazial- gebiet südlich der maximalen Kontinentalvereisung entwickeln und dort Grundlage der Bodenbildung sein konnte. Bei Zwickau und bei Chemnitz weicht die nördliche Arealgrenze in jeweils auffälliger Bucht deutlich südwärts zurück, was ebenfalls dem Verlauf der Feuersteinlinie entspricht. Dergestalt blieben Funde der Art im Rümpfwald bei Zwickau aus. Auch in der paarigen Chemnitzer Bucht wurde die Art vielerorts nicht nachgewiesen: So ist im südwestlichen Teil dieser Bucht z.B. der Steegenwald und nordwestliche Hauwald, andererseits im südöstlichen der Abtwald ohne bisherige Funde.

Das nordwestliche Stück der Arealgrenze der Art verlässt die Feuersteinlinie westlich der Weißen Elster, bei Weida, und nimmt seinen weiteren Verlauf im Ostthüringer Schiefergebirge in südwestlicher Richtung am Westrand des Vogtlandes, ungefähr bei Triebes, Zeulenroda, Schleiz, also am Rande der Ziegenrücker Mulde, und erreicht bei Burgk die Saale. Im südwestlichen Vogtland verläuft die Verbreitungsgrenze von der Saale nach Südosten, durch den Kämmerawald bei Tanna und ostwärts über das Eichigt/Birkigt bei Rößnitz bis zur Weißen Elster bei Plauen. Südwärts über Oelsnitz/ Vogtl. erreicht sie südlich von Triebel/ Vogtl. den Schirningwald und bei Rehau das nördliche Fichtelgebirge. Weiter südwärts durchquert die Arealgrenze das Fichtelgebirge und verläuft entlang des Oberpfälzer Waldes. Der von uns ermittelte westlichste deutsche Fundort der Art ist der Burgkwald an der Oberen Saale (4213). Dieser liegt ca. $45 \mathrm{~km}$ weiter westlich als jener, den Makeschin (1987) im Oberpfälzer Wald bei Waldsassen für den westlichsten hielt. Der nordwestlichste der bisherigen Fundpunkte liegt im Schömberger Wald bei Steinsdorf (4375), unweit Weida.

Auf den Talhängen der Zwickauer Mulde tritt die Regenwurmart auch wenig nördlicher als die Feuersteinlinie auf, so ein Stück weit im Gebiet, das vom Gletscherrand erreicht wurde: nördlich von Zwickau (4007, 4014), bei Glauchau (z.B. 3908, 3909, 3913, 3918, 3919), Waldenburg $(3920,3922-3927,4117,4118)$ und Penig (39103912, 4327, 4397, 4398). Diese Standorte gehören 
offenbar zu Bereichen, in denen viel westerzgebirgisches Verwitterungsmaterial im eiszeitlichen Elster-2-Stadium abgelagert wurde (Wolf \& Schubert 1992). Nach jüngster geologischer Kartierung (Alexowsky et al. 2007) befinden sich die zwei nördlich von Zwickau nachgewiesenen Vorkommen der Art $(4007,4014)$ auf tonigem, sandig-kiesigem, braungrauen Schluff des Weichselglazials $\left({ }_{d} Q W\right)$ über kryoturbatem elstereiszeitlichem Schluff (gQE2), von dem Altermann et al. (2008) zufolge wahrscheinlich beträchtliche Anteile in den Boden eingemischt wurden.

\section{Fundpunkt-Verteilung und Bodenausgangsgesteine}

Die Verteilung der Fundpunkte von D. illyrica hat auffällig enge Beziehung zur regionalen Verteilung der Bodenausgangsgesteine aus der Gruppe der sauren Magmatite und Metamorphite. Die meisten Fundpunkte befinden sich innerhalb der auf der Karte 2.1.1 in BGR (2016) dargestellten, ums Böhmische Massiv gruppierten großflächigen Verbreitungsgebiete dieser Ausgangsgesteine. Deutlich gehäuft sind diesbezügliche Fundpunkte am nordwestlichen Rand des Areals der Art, im inselartigen Gebiet des Vorkommens dieser Gesteine zwischen der Oberen Saale bei Schleiz und der Weißen Elster (Mühltroffer Wald, Schwandhölzer, Kettenwald, Köthenwald, Mehltheuerwald, Syrau-Kauschwitzer Heide, Eichigt). In geringerem Maße sind Funde der Art in Böden über Tongesteinen nachgewiesen (z.B. 4004 bei Rödlitz im Erzgebirgsbecken, 4304 bei Leubetha im Vogtland), wo diese an die Vorkommen saurer Magmatite und Metamorphite angrenzen. Daneben gibt es einzelne Funde in Böden über Sandsteinen (z.B. 3239 im Elbsandsteingebirge, 4168 bei Pöllwitz im Vogtland). Nicht angetroffen wurde die Art im Frankenwald, der im Tonschiefer-Gebiet liegt. Offenbar meidet sie das Gebiet basischer Magmatite und Metamorphite. So fehlt sie offensichtlich im Bayerischen Vogtland zwischen Hirschberg und Hof.

\section{Körperliche Ausprägung und Populationsstruktur der Art an den Fundorten}

Inmitten des Verbreitungsgebietes von $D$. illyrica wurden äußerlich vollausgeprägte, geschlechtsreife Individuen der Art nachgewiesen, deren männliche Poren des 15. Segments von erhabenen, mächtigen Drüsenhöfen umgeben sind, die beide benachbarte Segmente vollkommen einnehmen. Diesen segmentübergreifenden Drüsenhöfen fehlen die Intersegmentalfurchen. Die vollausgeprägten Tiere tragen auf dem 28. - 34. Segment einen drüsig verdickten, sattelförmigen Gürtel, Pubertätswälle am 31. - 33. und ventral am 29. oder 30. Segment extern adhäsive Spermatophoren. Ihr Anteil an der Gesamtmenge der im untersuchten Gebiet gesammelten erwachsenen Tiere beträgt $\sim 10$ Prozent. Sie stammen von $c a$. 20 Prozent der Fundpunkte. Nicht nachweisbar waren derartig ausgeprägte Individuen an zahlreichen Standorten am Rand des Verbreitungsgebietes der Art und in offensichtlich suboptimal ausgestatteten Habitaten, auch z.B. in instabilen Hanglagen (3910-3912) und an möglicherweise gestörten Standorten. Letzteres betrifft z.B. den Bereich der von Kratzsch (1843) genannten Wüstung Reiboldsgrün (4168) mitsamt der umgebenden, $c a .15 \mathrm{~km}^{2}$ großen Waldfläche, die im 17. Jahrhundert für längere Zeit entwaldet war.

Stattdessen wurden hier und in zahlreichen anderen Fällen am Arealrand große erwachsene Tiere der Art gefunden, die allesamt reduzierte äußere reproduktive Strukturen besitzen. Bei vielen Individuen dieser Gruppe treten die deutlichen männlichen Poren des 15. Segments aus einem kleinen flachen bis unauffälligen, aber hofartigen Wall hervor, der stets auf dieses Segment beschränkt ist. Manchmal ist dort ein verdickter Drüsenhof ausgeprägt, der dann aber stets die Segmente 14 und 16 nicht vollkommen einnimmt, zumeist als ein auf das 15. Segment aufgesetzter erscheint oder im Falle von mächtiger Haut- 
verdickung um den Porus am Rande des Drüsenhofs von den Intersegmentalfurchen durchzogen ist. Der Gürtel der Tiere dieser Gruppe ist nicht sattelförmig, sondern ein dünnerer, drüsiger Überzug, der im dafür arteigenen Bereich von Segmenten farblich (zumeist dunkler) abgesetzt ist. Bei einigen dieser Tiere ist die Ausdehnung des Gürtels kürzer, indem er das 28. Segment oder jeweils zwei Segmente (28 und 34 oder 33 und 34) nicht einbezieht. Zumeist sind vorhandene Pubertätswälle angedeutet, bei einigen Tieren fehlen sie. Die Fundpunkte, an denen ausschließlich Tiere mit derartig reduzierten äußeren reproduktiven Organen angetroffen wurden, deuten einen Kranz um das Kernareal der Art an.

Neben diesen abweichend ausgeprägten kamen in einzelnen Fällen unter den erwachsenen Tieren desselben Fundpunkts auch äußerlich vollausgeprägte vor (z.B. Neudörfler Wald 4040: 3 von 5; Eichlaide 4397: 1 von 5), die dann stets kleiner waren. Zumeist am Arealrand traten in der Population in überwiegender Mehrzahl Tiere ohne Gürtel und Drüsenhöfe, aber mit undeutlichem männlichen Porus auf, der meist vom genannten flachen hofartigen Wall umgeben ist. Sie überwogen in der Gesamtmenge der im untersuchten Gebiet gefundenen Individuen und waren vor allem große Tiere. Sehr kleine ohne Gürtel und männlichen Porushof, d.h. juvenile immature Individuen, traten an wenigen Bodenstandorten auch in Anhäufung auf (z.B. Langer Busch 3910, Schirningwald 4303).

\section{Habitat}

Alle Individuen der Art wurden als epigäische bis epi-endogäische Lebensformen im Waldboden gefunden, so im organischen Auflagehorizont (L, Of, Oh) und im anschließenden oberen Saum des Mineralischen Oberbodenhorizonts (Ah), der akkumulierten Humus enthält. Auch von Standorten mit geringem Bodenabtrag, teils schmalen Horizonten und temporärer Streuauflage in Hanglage liegen Nachweise der Art vor (z.B. Langer Busch 3910, Oberhang). Die vollausgeprägten, geschlechtsreifen Individuen wurden ausschließlich in Bodenprofilen mit vollständigem Horizont- spektrum angetroffen, so im Grenzbereich Feinhumus / Ah-Horizont unter geschlossener Streuschicht an Standorten mit stabilem Jahresgang von genug Feuchtigkeit, z.B. in grundfeuchten Böden von Tallagen, oder an der Unterseite von Moosdecken, also im Substrat, das sommerüber sehr lange Feuchtigkeit hält. Die Art kommt sowohl in Böden unter Laubstreu als auch in solchen unter Nadelstreu vor, zumeist auf basenarmen Braunerden (Cambisols) der Mittelgebirgslagen. Die vollausgeprägten geschlechtsreifen Indiiduen mit sattelförmigen Gürtel fehlten in den D. illyrica-Populationen der offensichtlich weniger günstigen Habitate (bei Hanglage, abweichendem Horizontspektrum, Mangel an Laubstreu).

\section{Funddaten}

Im Folgenden werden genannt: Nummer der Untersuchung, Anzahl determinierter Individuen (Ex.) außer juvenilen immaturen, Waldgebiet, örtliche Lage, Koordinaten des Fundorts, Höhenlage, dominierende Baumarten, Funddatum. Die Belege befinden sich in der Sammlung des Autors.

Böhmerwald/Bayerischer Wald: 4362: 1 Ex., Zwerchecker Wald, bei Oberhaiderberg, 4909'54"N, $13^{\circ} 08^{\prime} 26^{\prime \prime E}, 1010 \mathrm{~m}$, Fichten, Tannen, 10.08.2019. 4363: 1 Ex., Pfefferwald, bei Ottmannszell, 49¹0' $12^{\prime \prime N}, 13^{\circ} 00^{\prime} 14^{\prime \prime E}, 750 \mathrm{~m}$, Fichten, Tannen, 10.08. 2019.

Oberpfälzer Wald: 4366: 4 Ex., Glashüttner Wald, bei Altglashütte, $49^{\circ} 46^{\prime} 13^{\prime \prime N}, 12^{\circ} 22^{\prime} 47^{\prime \prime} \mathrm{E}, 749 \mathrm{~m}$, Fichten, Tannen, 17.08.2019. 4365: 1 Ex., Wald Altherrgott, bei Mähring, 49॰55'41"N, 12²9'37"E, $695 \mathrm{~m}$, Fichten, Tannen, Buchen, 17.08.2019. 4364: 1 Ex., Klosterwald Archenoe/Hammerholz, bei Pfaffenreuth, $49^{\circ} 58^{\prime} 14^{\prime \prime} \mathrm{N}, 12^{\circ} 18^{\prime} 38^{\prime \prime} \mathrm{E}, 538 \mathrm{~m}$, Fichten, Kiefern, Eichen, 17.08.2019.

Fichtelgebirge: 4367: 1 Ex., Bärenholz, bei Quellenreuth, 50¹3'57"N, 11 ${ }^{\circ} 59^{\prime} 03^{\prime \prime E}, 582 \mathrm{~m}$, Fichten, 24.08.2019. 4298: 3 Ex., Rehauer Wald, bei Sophienreuth, $50^{\circ} 13^{\prime} 27^{\prime \prime} \mathrm{N}, 12^{\circ} 05^{\prime} 28^{\prime \prime} \mathrm{E}, 560 \mathrm{~m}$, Fichten, 05.05.2018. 4207: 2 Ex., Dürrschachtwald am Großen Hengstberg, bei Silberbach, 5007'45"N, $12^{\circ} 10^{\prime} 56^{\prime \prime} \mathrm{E}$, 565 m, Fichten, Lärchen, 10.06.2017.

Elstergebirge: 4329: 1 Ex., Pechlohe, bei Raunergrund, $50^{\circ} 15^{\prime} 59^{\prime \prime} \mathrm{N}, 12^{\circ} 18^{\prime} 25^{\prime \prime} \mathrm{E}, 518 \mathrm{~m}$, Fichten, 24.11.2018. 
Vogtland: 4389: 2 Ex., Kämmerawald, bei Tanna, $50^{\circ} 28^{\prime} 13^{\prime \prime N}, 1^{\circ} 51^{\prime} 07^{\prime \prime E}, 584 \mathrm{~m}$, Fichten, 28.09.2019. 4174: 1 Ex., Oschitzer Wald, bei Heinrichsruh, 50 $32^{\prime} 23^{\prime \prime N}, 11^{\circ} 48^{\prime} 02^{\prime \prime E}, 552 \mathrm{~m}$, Fichten, 19.11.2016. 4213: 2 Ex., Burgkwald, bei Burgk, 50³2'31"N, $11^{\circ}$ 42'51"E, 423 m, Fichten, Buchen, 29.07.2017. 4405: 1 Ex. Schleiz-Oberböhmsdorfer Wald, bei Wüstendit-

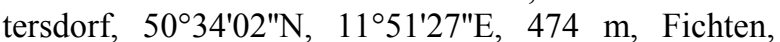
13.10.2019. 4273: 6 Ex., Leitenholz, bei Rödersdorf, $50^{\circ} 38^{\prime} 01^{\prime \prime} \mathrm{N}, 11^{\circ} 50 ' 24^{\prime \prime E}, 447 \mathrm{~m}$, Fichten, 04.11.2017. 4244: 2 Ex., Schwandhölzer, bei Dröswein, 50³5' $16^{\prime \prime} \mathrm{N}, 11^{\circ} 54^{\prime} 30^{\prime \prime E}, 479 \mathrm{~m}$, Fichten, Kiefern, 23.09.

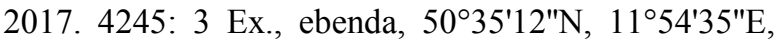
490 m, Fichten, Kiefern, 23.09.2017. 4224: 4 Ex., Kettenwald, bei Dröswein, 50³6'00"N, 11 ${ }^{\circ} 55^{\prime} 54^{\prime \prime} \mathrm{E}$, 438 m, Fichten, 20.08.2017. 4227: 4 Ex., Köthenwald, bei Grüngut, $50^{\circ} 36^{\prime} 34^{\prime \prime} \mathrm{N}, 11^{\circ} 56^{\prime} 13^{\prime \prime} \mathrm{E}, 435 \mathrm{~m}$, Fichten, 25.08.2017. 4376: 1 Ex., Pahrener Hölzer, bei Klein-

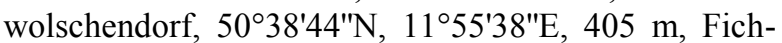
ten, Eichen, 11.09.2019. 4246: 7 Ex., Mühltroffer

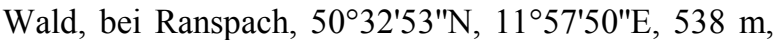
Fichten, Kiefern, 03.10.2017. 4247: 3 Ex., ebenda, $50^{\circ} 32^{\prime} 51^{\prime \prime N}, 11^{\circ} 57^{\prime} 42^{\prime \prime E}, 542 \mathrm{~m}$, Fichten, 03.10.2017. 4253: 1 Ex., Hammerholz, bei Demeusel, 50॰31'08"N, 11 ${ }^{\circ} 58^{\prime} 35^{\prime \prime E}, 462 \mathrm{~m}$, Fichten, Kiefern, 09.10.2017.

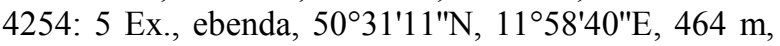
Fichten, Kiefern, 09.10.2017. 4383: 3 Ex., Eichigt, bei Rößnitz, 50॰30'01"N, 1201'20"E, $458 \mathrm{~m}$, Fichten, Kiefern, 18.09.2019. 4384: 1 Ex., ebenda, 50²9'49"N, $12^{\circ} 01 ' 27 " \mathrm{E}, 473 \mathrm{~m}$, Fichten, Kiefern, 18.09.2019. 4382: 1 Ex., Syrau-Kauschwitzer Heide, bei Schneckengrün, 50॰31'14"N, 1202'54"E, $527 \mathrm{~m}$, Fichten, Kiefern, 18.09.2019. 4125: 2 Ex., Mehltheuerwald, bei Syrau, 50³2'32"N, 1203'14"E, $512 \mathrm{~m}$, Fichten, 13.08.2016. 4255: 9 Ex., Reiboldsgrün, bei Leitlitz, $50^{\circ} 37^{\prime} 11^{\prime \prime N}, 11^{\circ} 59^{\prime} 17^{\prime \prime E}, 457 \mathrm{~m}$, Fichten, Kiefern, 09.10.2017. 4168: 1 Ex., Reiboldsgrün, bei Pöllwitz, $50^{\circ} 37^{\prime} 30^{\prime \prime} \mathrm{N}, 12^{\circ} 00^{\prime} 24^{\prime \prime} \mathrm{E}, 423 \mathrm{~m}$, Buchen, Fichten, 31.10.2016. 4226: 5 Ex., Pöllwitzer Wald, bei Pöllwitz, $50^{\circ} 37^{\prime} 24^{\prime \prime N}, 12^{\circ} 03^{\prime} 03^{\prime \prime E}, 419 \mathrm{~m}$, Fichten, Kiefern, 20.08.2017. 4162: 7 Ex., Pöllwitzer Wald, bei Wellsdorf, 50³7'21"N, 1205'23"E, 450 m, Kiefern, Fichten, 20.10.2016. 4413: 1 Ex., Metschwald, bei Niederböhmersdorf, 50³9'21"N, 1202'00"E, 428 m, Kiefern, Fichten, 23.10.2019. 4164: 1 Ex., Metschwald, bei Neuärgerniß, 50³9'27"N, 1203'01"E, 409 m, Fichten, Eichen, 28.10.2016. 4170: 5 Ex., Niederböhmersdorfer Wald, bei Mehla, 50 $40^{\prime} 43^{\prime \prime N}, 12^{\circ} 02^{\prime} 48^{\prime \prime E}, 410 \mathrm{~m}$, Fichten, Kiefern, 04.11.2016. 4375: 1 Ex., Schömberger Wald, bei Steinsdorf, 5045'06"N, 12 ${ }^{\circ} 01^{\prime} 57^{\prime \prime E}$, 374 m, Fichten, Kiefern, 11.09.2019. 4319: 1 Ex., Obere Harth, bei Langenwetzendorf, 50 $40^{\prime} 35^{\prime \prime} \mathrm{N}$, $12^{\circ} 07^{\prime} 06 " \mathrm{E}, 386$ m, Fichten, Erlen, 09.09.2018. 4372:
2 Ex., Bergaer Wald, bei Wernsdorf, 50 $46^{\prime} 10^{\prime \prime} \mathrm{N}$, $12^{\circ} 08^{\prime} 02 " \mathrm{E}, 335$ m, Fichten, Eichen, 04.09.2019. 4374: 2 Ex., Hofholz, bei Endschütz, 5046'29"N, $12^{\circ}$ 08'03"E, 325 m, Kiefern, Buchen, 11.09.2019. 4153: 1 Ex., Greizer Wald, bei Kleinreinsdorf, $50^{\circ} 42^{\prime} 18^{\prime \prime} \mathrm{N}$, $12^{\circ} 12^{\prime} 51 " \mathrm{E}, 315 \mathrm{~m}$, Fichten, Kiefern, 11.10.2016. 4048: 2 Ex., Greizer Wald, bei Waldhaus, 5041'45"N, $12^{\circ} 15^{\prime} 42^{\prime \prime E}, 400 \mathrm{~m}$, Fichten, Kiefern, 10.10.2015. 4049: 1 Ex., ebenda, 5041'52"N, 12॰14'55"E, 366 m, Fichten, Buchen, 22.10.2015. 4050: 8 Ex., ebenda, $50^{\circ} 41^{\prime} 55^{\prime \prime} \mathrm{N}, 12^{\circ} 14^{\prime} 49^{\prime} \mathrm{E}, 354 \mathrm{~m}$, Fichten, Kiefern, 22. 10.2015. 4044: 3 Ex., Greizer Wald, bei Teichwolframsdorf, $50^{\circ} 42^{\prime} 49^{\prime \prime} \mathrm{N}, 12^{\circ} 15^{\prime} 05^{\prime \prime} \mathrm{E}, 366 \mathrm{~m}$, Fichten, Buchen, 06.10.2015. 4275: 7 Ex., Trünziger Wald, bei

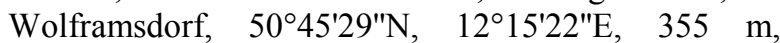
Fichten, Kiefern, 09.11.2017. 4218: 4 Ex., Trünziger Wald, bei Walddorf, $50^{\circ} 44^{\prime} 12^{\prime \prime} \mathrm{N}, 12^{\circ} 15^{\prime} 03^{\prime \prime} \mathrm{E}, 348 \mathrm{~m}$, Buchen, Kiefern, 07.08.2017. 4046: 12 Ex., Werdauer Wald, bei Reudnitz, 5041'18"N, 12०17'17"E, 368 m, Kiefern, Fichten, 10.10.2015. 4021: 2 Ex., Schönfelser Wald, bei Ebersbrunn, 50³9'14"N, 12²4'38"E, 408 m, Fichten, Buchen, 05.09.2015. 4393: 2 Ex., Herlasgrüner Wald, bei Helmsgrün, 50³3'21"N, 12 $2^{\circ} 13^{\prime} 27^{\prime \prime} \mathrm{E}$, 429 m, Fichten, Birken, Eichen, 03.10.2019. 4055: 2 Ex., Treuener Wald, bei Wolfspfütz, 50³3'36"N, 12०19'56"E, $454 \mathrm{~m}$, Fichten, Buchen, 07.11.2015. 4122: 4 Ex., ebenda, 50॰33'36"N, 12²0'01"E, 444 m, Fichten, Buchen, 13.08.2016. 4056: 2 Ex., Frohnholz, bei Altmannsgrün, 50³0'34"N, 12॰18'54"E, $443 \mathrm{~m}$, Fichten, 07.11.2015. 4057: 1 Ex., Mauerholz, bei Altmannsgrün, 50³0'14"N, 12 $16^{\prime} 55^{\prime \prime} \mathrm{E}, 460 \mathrm{~m}$, Fichten, Lärchen, 07.11.2015. 4424: 2 Ex., Jägerswald, bei Tirpersdorf, $50^{\circ} 26^{\prime} 38^{\prime \prime N}$, $12^{\circ} 15^{\prime} 27^{\prime \prime} \mathrm{E}, 570 \mathrm{~m}$, Fichten, 08.11.2019. 4330: 1 Ex., Görnitzholz, bei Raasdorf, $50^{\circ} 23^{\prime} 47^{\prime \prime N}, 12^{\circ} 12^{\prime} 47^{\prime \prime E}, 435$ m, Fichten, Kiefern, 24. 11.2018. 4304: 15 Ex., Tännicht, bei Leubetha, 50²0' $50 " \mathrm{~N}, 12^{\circ} 15^{\prime} 27^{\prime \prime E}, 470 \mathrm{~m}$, Fichten, Kiefern, 19.05. 2018. 4308: 2 Ex., Schirningwald, bei Süßebach, $50^{\circ} 21^{\prime} 06 " \mathrm{~N}, 12^{\circ} 09^{\prime} 24^{\prime \prime E}, 580 \mathrm{~m}$, Fichten, Kiefern, 09.06.2018. 4303: 11 Ex., Schirningwald, bei Tiefenbrunn, $50^{\circ} 20^{\prime} 01^{\prime \prime} \mathrm{N}, 12^{\circ} 08^{\prime} 08^{\prime \prime E}, 612 \mathrm{~m}$, Fichten, Kiefern, 19.05.2018. 4302: 7 Ex., Rehauer Wald, bei Ludwigsbrunn, 50¹7'06"N, 12 $06^{\prime} 13^{\prime \prime E}, 615$ m, Fichten, Kiefern, 19.05.2018. 4371: 1 Ex., Löwitz, bei Rehau, $50^{\circ} 16^{\prime} 11^{\prime \prime} \mathrm{N}, 12^{\circ} 02^{\prime} 50 " \mathrm{E}, 576 \mathrm{~m}$, Fichten, Kiefern, Buchen, 24.08.2019.

Mulde-Lösshügelland: 1051: 1 Ex., Langer Busch, bei Wolperndorf, 5054'53"N, 12 ${ }^{\circ} 38^{\prime} 44^{\prime \prime} \mathrm{E}, 265 \mathrm{~m}$, Hainbuchen, Linden, 22.05.1991. 3910: 6 Ex., ebenda, 02.11.2014. 3912: 1 Ex., ebenda, 50 $54^{\prime} 33^{\prime \prime} \mathrm{N}, 12^{\circ} 38^{\prime}$ 28"E, 240 m, Birken, Linden, 02.11.2014. 1048: 3 Ex., ebenda, 50॰54'15"N, 12॰38'28"E, 225 m, Erlen, Linden, 11.05.1991. 3911: 4 Ex., ebenda, 02.11.2014. 
4327: 2 Ex., Buchholz, bei Kaufungen, 5054'14"N, 12॰41'22"E, $252 \mathrm{~m}$, Fichten, Lärchen, 09.11.2018. 4121: 3 Ex., Jahnshorn, bei Niederfrohna, 50 $53^{\prime} 00^{\prime \prime} \mathrm{N}$, 12 ${ }^{\circ} 43^{\prime} 06^{\prime \prime E}, 307 \mathrm{~m}$, Eichen, Birken, 11.08.2016. 4151: 1 Ex., Tümmel, bei Pleißa, 5049'35"N, 1244'36"E, 418 m, Fichten, Lärchen, 07.10.2016. 4115: 1 Ex., Oberwald, bei Langenberg, 50॰49'24"N, 1243'17"E, 460 m, Fichten, Eichen, 18.07.2016. 3920: 1 Ex., Callenberger Kirchenholz, bei Callenberg, 50 $50^{\prime} 44^{\prime \prime} \mathrm{N}$, 12॰37'33"E, 288 m, Buchen, Eichen, 20.11.2014. 3922: 1 Ex., Callenberger Holz, bei Oberwinkel, 50 $51^{\prime} 03^{\prime \prime} \mathrm{N}$, 12³7'21"E, 320 m, Buchen, Birken, 20.11.2014. 39233926: 12 Ex., Callenberger Holz, bei Naundorf, $50^{\circ}$ $51^{\prime} 29^{\prime \prime N}, 12^{\circ} 37^{\prime} 54^{\prime \prime E}, 328 \mathrm{~m}$, Eichen, Buchen, 23.11. 2014. 3927: 4 Ex., ebenda, 50॰51'32"N, 12³7'45"E, 334 m, Buchen, Fichten, 23.11.2014. 4117: 1 Ex., Waldenburger Stadtwald, bei Langenchursdorf, 50 $52^{\prime}$ $22^{\prime \prime N}, 12^{\circ} 37^{\prime} 57^{\prime \prime E}, 280$ m, Fichten, Eichen, 07.08.2016. 4118: 3 Ex., ebenda, 5052'25"N, 12॰38'06"E, $302 \mathrm{~m}$, Eichen, Kiefern, 07.08.2016. 4358: 14 Ex., Gersdorfer Wald, bei Remse, 5051'22"N, 12³5'20"E, $235 \mathrm{~m}$, Fichten, 21.06.2019. 3908,3918,3919: 8 Ex., Klosterholz, bei Weidensdorf, 5051'45"N, 12³2'17"E, 275 m, Erlen, Eichen, 31.10. und 11.11.2014. 3909: 1 Ex., ebenda, 50 $51^{\prime} 43^{\prime \prime} \mathrm{N}, 12^{\circ} 32^{\prime} 25^{\prime \prime} \mathrm{E}, 282 \mathrm{~m}$, Buchen, 31.10.2014. 1070: 1 Ex., ebenda, $50^{\circ} 51^{\prime} 39^{\prime \prime} \mathrm{N}, 12^{\circ} 32^{\prime}$ 42"E, 292 m, Birken, Eichen, 19.06.1991. 3913: 1 Ex., ebenda, 03.11.2014. 1071: 1 Ex., Klosterholz, bei Kleinchursdorf, 5051'31"N, 12 ${ }^{\circ} 33^{\prime} 07^{\prime \prime E}, 283$ m, Erlen, Eichen, 19.06.1991. 4397: 5 Ex., Eichlaide, bei Dürrenuhlsdorf, 5053'14"N, 12॰36'56"E, $275 \mathrm{~m}$, Fichten, 06.10.2019. 4398: 3 Ex., ebenda, 50 $53^{\prime} 12^{\prime \prime} \mathrm{N}, 12^{\circ} 37^{\prime}$ 02"E, $281 \mathrm{~m}$, Buchen, 06.10.2019.

Erzgebirgsbecken: 4007: 2 Ex., Harthwald, bei Mosel, 5046'48"N, 12²6'47"E, 296 m, Eichen, Lärchen, 05.08.2015. 4014: 3 Ex., Schäbigtwald, bei Kalthau-

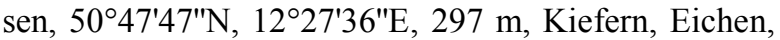
23.08.2015. 4149: 1 Ex., Rabensteiner Wald, bei Grüna, 50॰48'58"N, 12॰45'55"E, $418 \mathrm{~m}$, Fichten, Buchen, 04.10.2016. 4150: 3 Ex., ebenda, 5049'01"N, 12॰45'43"E, 432 m, Fichten, 07.10.2016. 4004: 3 Ex., Buchwald, bei Rödlitz, 5043'23"N, 1240'29"E, 453 m, Fichten, Kiefern, Buchen, 31.07.2015. 3998: 2 Ex., Neudörfler Wald, bei Neuschönburg, 5042'42"N, 12³9'33"E, $433 \mathrm{~m}$, Fichten, Kiefern, 14.07.2015. 4041: 1 Ex., Lichtensteiner Stadtwald, bei Lichtenstein/ Sa., 5045'41"N, 12³9'09"E, 338 m, Erlen, Birken, 30.09.2015. 4030: 1 Ex., Burgwald, bei Heinrichsort, $50^{\circ} 44^{\prime} 02^{\prime \prime} \mathrm{N}, 12^{\circ} 37^{\prime} 42^{\circ} \mathrm{E}, 380 \mathrm{~m}$, Fichten, Lärchen, 21.09.2015. 4001: 2 Ex., Zschockener Holz, bei Zschocken, 50³9'38"N, 12³8'08"E, 396 m, Fichten, Buchen, 28.07.2015. 4107: 7 Ex., Vielauer Wald, bei Vielau, 50 $41^{\prime} 04^{\prime \prime} \mathrm{N}, 12^{\circ} 31^{\prime} 36^{\prime \prime} \mathrm{E}, 329 \mathrm{~m}$, Eichen,
Birken, 27.06.2016. 4152: 1 Ex., Werdauer Wald, bei Langenbernsdorf, $50^{\circ} 44^{\prime} 02^{\prime \prime} \mathrm{N}, 12^{\circ} 18^{\prime} 43^{\prime \prime} \mathrm{E}, 331 \mathrm{~m}$, Fichten, Buchen, 09.10.2016. 4154: 2 Ex., Kleiner Wald, bei Langenbernsdorf, 50 $43^{\prime} 58^{\prime \prime} \mathrm{N}, 12^{\circ} 16^{\prime} 11^{\prime \prime} \mathrm{E}$, 346 m, Buchen, Kiefern, 11.10.2016.

Westerzgebirge: 3970: 1 Ex., Unterer Wiesenburger Wald, bei Hartmannsdorf b. Kirchberg, $50^{\circ}$ $35^{\prime} 40^{\prime \prime} \mathrm{N}, 12^{\circ} 33^{\prime} 38^{\prime \prime} \mathrm{E}, 483 \mathrm{~m}$, Fichten, Eichen, 31.05. 2015. 3971: 9 Ex., Unterer Wiesenburger Wald, bei Saupersdorf, 50³6'16"N, 12³3'25"E, $441 \mathrm{~m}$, Birken, Eichen, 31.05.2015. 4113: 1 Ex., Kirmesmoos, bei Jägerhaus, $50^{\circ} 31^{\prime} 00^{\prime \prime} \mathrm{N}, 12^{\circ} 42^{\prime} 42^{\prime \prime E}, 755 \mathrm{~m}$, Fichten, Buchen, 16.07.2016. 4112: 3 Ex., Friedrichsheide, bei Erlabrunn, 50²8'54"N, 12॰42'29"E, 725 m, Fichten, Buchen, 16.07.2016. 4111: 1 Ex., Wald am Auersberg, bei Sauschwemme, $50^{\circ} 26^{\prime} 23^{\prime \prime N}, 12^{\circ} 40^{\prime} 15^{\prime \prime E}, 842 \mathrm{~m}$, Fichten, 16.07.2016. 4110: 3 Ex., Wintergrün, bei Carlsfeld, 50²6'32"N, 12³5'15"E, 916 m, Fichten, 16.07.2016. 4102: 1 Ex., Pyrawald, bei Sachsengrund, $50^{\circ} 25^{\prime} 01^{\prime \prime} \mathrm{N}, 12^{\circ} 32^{\prime} 27^{\prime \prime E}, 830 \mathrm{~m}$, Fichten, 11.06.2016. 4103: 1 Ex., ebenda, 50²4'55"N, 12³2'27"E, $863 \mathrm{~m}$, Fichten, Buchen, 11.06.2016. 4101: 5 Ex., Riedert, bei Wilzschhaus, 50²8'02"N, 12³1'40"E, $630 \mathrm{~m}$, Tannen, Fichten, 11.06.2016. 4098: 3 Ex., Grüner Wald, bei Hammerbrücke, $50^{\circ} 27^{\prime} 14^{\prime \prime} \mathrm{N}, \quad 12^{\circ} 24^{\prime} 17^{\prime \prime} \mathrm{E}, 706 \mathrm{~m}$, Buchen, Fichten, 28.05.2016. 4097: 3 Ex., Heroldswald, bei Muldenberg, 50²5'41"N, 12²2'29"E, 730 m, Fichten, 28.05.2016.

Mittelerzgebirge: 4040: 5 Ex., Neudörfler Wald, bei Ortmannsdorf, 5041'09"N, 12³7'46"E, $412 \mathrm{~m}$, Fichten, Birken, 30.09.2015. 4434: 1 Ex., Heiliger Wald, bei Mitteldorf,5041'21"N, 12 ${ }^{\circ} 47^{\prime} 17^{\prime \prime} \mathrm{E}, 505 \mathrm{~m}$, Kiefern, Fichten , Birken, 24.11.2019. 4106: 1 Ex., Hauwald, bei Jahnsdorf/ Erzgeb., 50 43'24"N, $12^{\circ}$ 50'29"E, 510 m, Fichten, Buchen, 20.06.2016. 4429: 2 Ex., Lohwald, bei Gornsdorf, 5041'28"N, 12 ${ }^{\circ} 52^{\prime} 48^{\prime \prime} \mathrm{E}$, 524 m, Fichten, 16.11.2019. 4407: 2 Ex., Kemtauer Wald, bei Kemtau, 5044'17"N, 12 ${ }^{\circ} 58^{\prime} 34^{\prime \prime} \mathrm{E}, 402 \mathrm{~m}$, Fichten, 17.10.2019. 4408: 3 Ex., Einsiedler Wald, bei Altenhain, 50॰46'46"N, 12 ${ }^{\circ} 59^{\prime} 20^{\prime \prime} \mathrm{E}, 450 \mathrm{~m}$, Fichten, 17.10.2019. 4210: 2 Ex., Struth, bei Erdmannsdorf, $50^{\circ} 49^{\prime} 52^{\prime \prime N}, 13^{\circ} 04^{\prime} 25^{\prime \prime} \mathrm{E}, 307 \mathrm{~m}$, Fichten, Kiefern, 08.07.2017. 4430: 1 Ex., Heinzewald, bei Heinze-

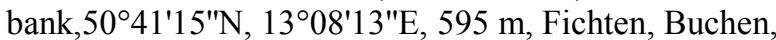
16.11.2019. 4418: 1 Ex., Geyerscher Wald, bei Jahnsbach, 50³9'00"N, 12॰55'16"E, $665 \mathrm{~m}$, Fichten, 04.11. 2019. 4419: 7 Ex., ebenda, 50³8'55"N, 12 ${ }^{\circ} 54^{\prime} 56^{\prime \prime} \mathrm{E}$, 642 m, Fichten, Buchen, 04.11.2019. 3989: 1 Ex., Geyerscher Wald, bei Dorfchemnitz, $50^{\circ} 39^{\prime} 08^{\prime \prime} \mathrm{N}$, 12॰51'10"E, 570 m, Fichten, 27.06.2015. 3876: 1 Ex., Hartensteiner Wald, bei Raum, 50 $39^{\prime} 02^{\prime \prime} \mathrm{N}, 12^{\circ} 42^{\prime}$ 10"E, 506 m, Fichten, Buchen, 27.09.2014.

Osterzgebirge: 4400: 2 Ex., Viehwegbusch, bei Bockendorf, $50^{\circ} 55^{\prime} 03^{\prime \prime} \mathrm{N}, 13^{\circ} 08^{\prime} 44^{\prime \prime} \mathrm{E}, 413 \mathrm{~m}$, Fichten, 
Kiefern, Birken, 11.10.2019. 4347: 4 Ex., Tharandter Wald, bei Grund, 5059'49"N, 13²8'47"E, 326 m, Kiefern, Buchen, 27.04.2019. 4344: 2 Ex., Tharandter

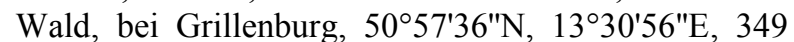
m, Fichten, Eichen, 27.04.2019. 4345: 1 Ex., Tharandter Wald, bei Kurort Hartha, 50 $58^{\prime} 03^{\prime \prime} \mathrm{N}, 13^{\circ} 31^{\prime}$ 24"E, 340 m, Fichten, 27.04.2019. 4426: 7 Ex., Dippoldiswalder Heide, bei Karsdorf, 50॰55'38'N, $13^{\circ} 41^{\prime}$ 29"E, 362 m, Fichten, 10.11.2019. 4427: 5 Ex., ebenda, $50^{\circ} 55^{\prime} 39^{\prime \prime} \mathrm{N}, 13^{\circ} 41^{\prime} 14^{\prime \prime E}, 374 \mathrm{~m}$, Kiefern, Fichten, 10.11.2019. 4350: 1 Ex., Vorderer Grünwald, bei Rehefeld-Zaunhaus, 5044'59"N, 1342'15"E, $812 \mathrm{~m}$, Fichten, Buchen, 11.05.2019. 4351: 4 Ex., Töpferwald, bei Neuhermsdorf,5044'17"N, 1338'13"E, 767 m, Fichten, Buchen, 11.05.2019.

Sächsische Schweiz: 3239: 1 Ex., Mittlerer Beutwald, bei Rosenthal, 5050'24"N, 14 01'05"E, $442 \mathrm{~m}$, Fichten, Buchen, 02.10.2011.

\section{DISKUSSION}

\section{Areal, Arealgrenze, Feuersteinlinie und Bodenausgangsgesteine}

Unser Beitrag belegt durch einwandfreie faunistische Unterlagen für 126 Fundorte, dass $D$. illyrica im untersuchten Gebiet weiter verbreitet ist, als bisher bekannt war. Unsere Funde umreißen den nordwestlichen Teil des Areals der Art, erhellen ihn aufgrund der Methodik aber nicht flächendeckend. Der Kartierungsmaßstab und die Trefferquote unserer Untersuchungen erlauben dennoch anzunehmen, dass diese Art ein kontinuierliches Areal auf dem östlichen Teil der Mitteleuropäischen Mittelgebirgsschwelle besiedelt. Dieses Areal liegt in jenem Gebiet, das vor Beginn der historischen Zeit von natürlichen Buchenwäldern der montanen Höhenstufen mit Tanne und Fichte eingenommen wurde (Firbas 1949, Walter \& Straka 1970, Breckle \& Agachanjanz 1994).

Da die ermittelte Nordgrenze des Areals von D. illyrica annähernd der Feuersteinlinie entspricht, ist diese Regenwurmart in ihrer Verbreitung auf ein geographisches Gebiet beschränkt, das seit dem Präelsterglazial eine ortsbürtige Kaltzeit-Verwitterungsdecke trägt (Büdel 1981), auf der sich über sehr lange Zeit relativ konti- nuierlich Boden entwickelte. Die epigäische $D$. illyrica bevorzugt im Bodenprofil den Grenzbereich von Auflagehumus und schwach humosem Mineralhorizont, ist aber offensichtlich von der über lange Zeit entstandenen lithogenen mineralischen Grundausrüstung des Bodens abhängig. Denn diese Art wurde in jenen Gebieten nicht vorgefunden, wo der elstereiszeitliche Gletscher die Verwitterungs- und Bodendecke ausgeräumt hatte oder diese als kryoturbat bis gravitativ zu Tal gleitendes periglaziales Material beim Eisrückschmelzen vollständig fluvial abgeführt wurde. Die von $D$. illyrica besiedelten Böden besitzen offenbar hohe Anteile von Kaltzeit-Verwitterungsprodukten, die im Zuge periglazialer Prozesse wie Kryoturbation und Kryotranslokation (Altermann et al. 2008) als Feinboden oder Bodenskelett in die Bodendecke eingemischt wurden. Zwischen zwei Eiszungen des Elster-1-Gletschers blieben die Verwitterungs- und Bodendecken offensichtlich weitgehend erhalten, wofür die illyrica-Vorkommen im Lohwald und östlichen Hauwald $(4429,4106)$ sprechen.

Das Areal von D. illyrica endet an seiner Nordwestgrenze mit der fichtelgebirgisch-erzgebirgischen Vorherrschaft (BGR 2016) der sauren Magmatite und Metamorphite als siliziumreiche Bodenausgangsgesteine. Dieses und die für regionale Stenözie sprechende Häufung von Funden am nordwestlichen Rand des Art-Areals, zwischen Oberer Saale und Weißer Elster, deuten wir als Zeichen einer engen Beziehung dieser Regenwurmart zu Verwitterungsprodukten aus diesen Gesteinen. Dem entspricht auch das Verschwinden der Art beim Übergang zur Ziegenrücker Mulde, wo höhere Anteile basischer Gesteine verwitterten. Die Verteilung der Art im untersuchten Gebiet belegt auch vereinzelte Vorkommen in Böden über angrenzenden Tongesteinen und Sandsteinen. Das gründet sich wohl auf die Tatsache, dass die Böden in den seltensten Fällen unmittelbar die Verwitterungsprodukte der am Standort selbst im Untergrund anstehenden Gesteine sind (Völkel et al. 2002). Die Böden des untersuchten Gebiets entwickelten sich in lithogenen Deckschichtprofilen, die aus jenen Gesteinen und/oder Böden entstanden, die im peri- 
glazialen Milieu der Standort-Umgebung umgebildet wurden. Die Schichten (Lagen) dieser Profile, so auch die allgemein oberflächenbildende Hauptlage, sind das Ergebnis von Turbationen und lateralen Umlagerungen, insbesondere von solifluidalem Ab- und Auftrag, und bestehen aus einem Lokalanteil, dem Verwitterten des liegenden Gesteins, und (z.B. äolischen) Fremdanteilen (Altermann 1993). Insofern variiert der unzweifelhaft bestehende Zusammenhang von stofflicher Zusammensetzung der Böden und dem liegendem Gestein des Standorts und stellt somit einen Valenzgradienten für $D$. illyrica dar.

Die am Talhang der Zwickauer Mulde nördlich der Feuersteinlinie, also im Randbereich maximaler Elstereisverbreitung, nachgewiesenen Vorkommen von D. illyrica (Schäbigtwald, Harth bei Mosel, Klosterholz, Langer Busch, Buchholz bei Kaufungen) deuten wir als Zeichen von Ablagerungen, die im Elster-2-Stadium erfolgten, aus der präelsterglazial begonnenen westerzgebirgischen Kaltzeit-Verwitterungsdecke stammen (Alexowsky et al. 2007, Wolf \& Alexowsky 2008) und an der Bildung rezenten Bodens teilnahmen. Wahrscheinlich hat dieses Material (gQE2) trotz Umlagerung die für D. illyrica wichtigen Eigenschaften der mineralischen Grundausrüstung bewahrt. Auch diese Beobachtung spricht für die Bindung der Regenwurmart an Faktoren aus der Verwitterungsdecke, die sich seit dem Praeelsterglazial entwickelte.

Mit dem Verlust der präelsterglazialen Verwitterungsdecke durch Vollabtrag nördlich der Feuersteinlinie im Verlaufe des Elsterglazials fiel offenbar die Grundlage einer Bodenentwicklung weg, die nicht nur für $D$. illyrica, sondern für viele Regenwurmarten lebensnotwendig ist. Das halten wir für die Ursache der von Michaelsen (1902) und Julin (1950) vorgestellten nördlichen Verbreitungsgrenze der endemischen Lumbriciden-Arten in der Holarktischen Region.

\section{Parthenogenese, Selektionstypen, Metapopulationen}

Die von uns gefundenen sichtlich erwachsenen Individuen von D. illyrica, die im Hinblick auf die schmaleren Drüsenhöfe ihrer männlichen Poren und auf die Pubertätswälle als äußerlich nicht voll ausgeprägte erscheinen, einen nicht sattelförmigen Gürtel und in einigen Fällen einen verkürzten Gürtel besitzen, stellen wir zur parthenogenetischen Form der Art. Den parthenogenetischen Regenwürmern fehlen bekanntlich in verschiedenen Graden einige sekundäre sexuelle Organe und morphologische Merkmale (Sims \& Gerard 1985, Edwards \& Bohlen 1996). Als Indikatoren für Parthenogenese betrachten wir das Fehlen der Pubertätswälle (Gates 1972, Hartenstein et al. 1980, Christian \& Zicsi 1999) und der drüsigen Hautverdickungen im männlichen Porushof, dessen dann unscheinbares Bild zumindest auf Retention der Vas deferens in einem jugendlichen $\mathrm{Zu}$ stand und damit auf männliche Sterilität hindeutet. Der sattelförmige Gürtel kennzeichnet die sexuelle, Spermatophoren bildende Form der Art (Zicsi 1965).

Den am untersuchten Arealrand der D. illyrica angedeuteten Kranz aus Fundpunkten wahrscheinlich parthenogenetischer Individuen halten wir für das Zeichen geographischer Parthenogenese (Vandel 1928). In solchem Falle besetzen die sich geschlechtlich fortpflanzenden Individuen den zentralen Bereich des Areals der Art, während die parthenogenetischen überwiegend am Arealrand vorkommen. Diese geographische Verteilung der beiden Fortpflanzungsformen wurde z.B. auch bei Diplopoden und Schaben festgestellt (Enghoff 1994, dort Fig. 2, bzw. Knebelsberger \& Bohn 2003, dort Fig. 6).

Parthenogenetische Individuen sind besser an unvorteilhafte Umweltbedingungen angepasst (Vandel 1940), z.B. an die kurzlebigen, an der Bodenoberfläche fleckig verteilten Ressourcen (Streu usw.), die sie mittels hoher Ansiedlungsfähigkeit und raschem Populationswachstum bei r-Selektion erfolgreich ausnützen. Demgegenüber herrscht im mineralischen Oberboden größere räumliche Kontinuität und zeitliche Stabilität, charakteristisch für ein Habitat, in dem KSelektion zu erwarten ist (Jaenike \& Selander 1979). Bei D. illyrica fallen Zeichen beider Selektionstypen auf: Die Art wird der epigäischen 
Lebensform zugeordnet (Pižl 2002a); wir fanden am Arealrand Habitate, in denen wie in der Periode aktiver Vermehrung (Edwards \& Lofty 1977) die immaturen die adulten Individuen anzahlmäßig stark überwogen (z.B. 3910, 4303), so auf wahrscheinliche r-Selektion hindeutend.

In suboptimalen Habitaten, vor allem am Arealrand, stellten wir überwiegend der parthenogenetischen Form zugeordnete Individuen fest. Eine ebensolche Relation in der Gesamtmenge der gesammelten erwachsenen Individuen erklärt sich aus der Nähe des untersuchten Gebietes zum Arealrand. Andererseits ermittelten wir im Arealinneren Fundpunkte von vollausgeprägten Individuen der Art. Bösener (1964) traf D. illyrica in der Nähe des Arealrands (Tharandter Wald) ,nie im Mineralboden“, nur in der Humusauflage der Waldbestände an. Im arealweiten Blickwinkel von Zicsi (1965) lebt die Art „,in der Laubstreu und in der obersten Bodenschicht". Unseren Beobachtungen zufolge tritt sie im Horizontbereich von Auflagehumus und oberflächennächstem Mineralboden auf. Das deutet eingedenk der oben angenommenen geographischen Parthenogenese darauf hin, dass D. illyrica möglicherweise Metapopulationen bildet, die aus parthenogenetischen und geschlechtlichen Unterpopulationen bestehen, wobei die parthenogenetischen besonders die Streu und den Auflagehumus nutzen, während die geschlechtlichen den obersten humosen Mineralboden bevorzugen. Einer Hypothese von Haag \& Ebert (2004) und unseren Beobachtungen zufolge erwarten wir derartige Metapopulationen in suboptimalen Habitaten und am Arealrand, wo weniger günstige Bedingungen herrschen und die Art vermutlich im häufigen Wechsel von lokaler Auslöschung und Wiederbesiedlung steht. Dabei sind die parthenogenetischen Unterpopulationen aufgrund ihrer körperlichen Verfassung in der Lage, durch ihre größere Dynamik (r-Strategie) den Bestand dieser Art zu erhalten.

Danksagung - Dr. Csaba Csuzdi unterstützte mich mit Hinweisen und kritischen Anmerkungen. Für technische Assistenz bei den Arbeiten im Freiland danke ich Frau Elke Höser und Herrn Michael Höser. Die Übersetzung ins Englische übernahm dankenswerterweise Herr Brian Hillcoat (Berlin).

\section{LITERATUR}

Alexowsky, W., Berger, H.-J., Goth, K., HÜBNER, F., Junghanns, C., Schneider, J.W. \& Wolf, L. (2007): Geologische Karte des Freistaates Sachsen 1: 25 000: Erläuterungen zu den Blättern 5240 Zwickau und 5241 Zwickau Ost. Sächsisches Landesamt für Umwelt und Geologie, Freiberg, 184 pp.

AltermanN, M. (1993): Gliederung von pleistozänen Lagen. Mitteilungen der Deutschen Bodenkundlichen Gesellschaft, 72: 825-828.

Altermann, M., JÄGER, K.-D., KopP, D., KowalKOWSKI, A., KÜHN, D. \& SCHWANECKE, W. (2008): Zur Kennzeichnung und Gliederung von periglaziär bedingten Differenzierungen in der Pedosphäre. Waldökologie, Landschaftsforschung und Naturschutz, 6: 5-42.

BGR Bundesanstalt für Geowissenschaften und Rohstoffe (2016): Bodenatlas Deutschland. Böden in thematischen Karten. BGR, Hannover. 144 pp.

BÖSENER, R. (1964): Die Lumbriciden des Tharandter Waldes. Zoologische Abhandlungen des Staatlichen Museums für Tierkunde Dresden, 27(9): 193-263.

BÖSENER, R. (1965): Untersuchungen über das Vorkommen und die forstliche Bedeutung von Lumbriciden (Oligochaeta) in verschiedenen Waldbeständen des Osterzgebirges. Wissenschaftliche Zeitschrift der Technischen Universität Dresden, 14(3): 741-746.

Breckle, S.-W. \& Agachanjanz, O. (1994): Spezielle Ökologie der Gemäßigten und Arktischen Zonen Euro-Nordasiens. 2. Auflage. Fischer, Stuttgart, Jena, 726 pp.

BÜDEL, J. (1981): Klima-Geomorphologie. 2. Auflage. Gebrüder Borntraeger, Berlin, Stuttgart. 304 pp.

ChristiAn, E. \& ZiCSI, A. (1999): Ein synoptischer Bestimmungsschlüssel der Regenwürmer Österreichs (Oligochaeta: Lumbricidae). Bodenkultur, 50(2): 121-131.

CognetTi, L. (1906): Nuovi dati sui Lumbricidi dell' Europa orientale. Bollettino dei Musei di zoologia ed anatomia comparata della $R$. Università di Torino, 21(527): 1-18.

Csuzdi, Cs., Pop, V.V. \& PoP, A.A. (2011): The earthworm fauna of the Carpathian Basin with new records and description of three new species (Oli- 
gochaeta: Lumbricidae). Zoologischer Anzeiger, 250(1): 2-18. doi: 10.1016/j.jcz.2010.10.001

DE LAtTIN, G. (1967): Grundriß der Zoogeographie. Gustav Fischer, Stuttgart, 602 pp.

Edwards, C.A. \& Bohlen, P.J. (1996): Biology and ecology of earthworms. Third Edition. Chapman \& Hall, London, 426 pp.

EDWARDS, C.A. \& LOFTY J.R. (1977): Biology of earthworms. Second Edition. Chapman and Hall, London, $333 \mathrm{pp}$.

EHLERS, J. (2011): Das Eiszeitalter. Spektrum Akademischer Verlag, Heidelberg, 363 pp.

EISSMANN, L. (1994): Grundzüge der Quartärgeologie Mitteldeutschlands. Altenburger naturwissenschaftliche Forschungen, 7: 55-135.

ENGHOFF, H. (1994): Geographical parthenogenesis in millipedes (Diplopoda). Biogeographica, 70(1): 25-31.

FIRBAS, F. (1949): Spät- und nacheiszeitliche Waldgeschichte Mitteleuropas nördlich der Alpen. Band 1: Allgemeine Waldgeschichte. Gustav Fischer, Jena, 480 pp.

FreitaG, H. (1962): Einführung in die Biogeographie von Mitteleuropa unter besonderer Berücksichtigung von Deutschland. Gustav Fischer, Stuttgart, $214 \mathrm{pp}$.

Gates, G.E. (1972): Burmese Earthworms. An introduction to the systematics and biology of megadrile oligochaetes with special reference to Southeast Asia. Transactions of American Philosophical Society, N. S., 62(7): 1-326.

HAAG, C.R. \& EBERT, D. (2004): A new hypothesis to explain geographic parthenogenesis. Annales Zoologici Fennici, 41: 539-544.

Hartenstein, R., Neuhauser, E.F. \& Easton, E.G. (1980): Growth and fecundity of $\mathrm{F}_{2}$ Eisenia foetida derived from $\mathrm{F}_{1} \mathrm{~s}$ both reared in isolation from birth. Megadrilogica, 3(11): 185-187.

JAENIKE, J. \& SELANDER, R.K. (1979): Evolution and ecology of parthenogenesis in earthworms. American Zoologist, 19(3): 729-737. doi: $10.1093 / \mathrm{icb} / 19.3 .729$

JuLin, E. (1950): De svenska daggmaskarterna. Arkiv för Zoologi, 42(17): 1-58.

KneBelsberger, T. \& BoHn, H. (2003): Geographic parthenogenesis in the subaptera-group of Phyllo- dromica (Blattoptera, Blattellidae, Ectobiinae). Insect Systematics \& Evolution, 34(4): 427-452. doi: $\underline{10.1163 / 187631203 X 00054}$

Kollmannsperger, F. (1936): Die von Prof. DAHL gesammelten Lumbriciden des Berliner Zoologischen Museums. Sitzungsberichte der Gesellschaft naturforschender Freunde Berlin, Jg. 1936 (3): 373-410.

KRATZSCH, J.F. (1843): Neuestes und gründlichstes Alphabetisches Lexicon der sämmtlichen Ortschaften der Deutschen Bundesstaaten. Erste Abtheilung. Verlag Eduard Zimmermann, Naumburg. 827 pp. [p. 560].

KulA, E. \& Švarc, P. (2012): Earthworms (Lumbricidae) from a surface layer and wireworms (Elateridae) of forest stands in the anthropogenically-disturbed area of the Děčínská vrchovina Upland (Czech Republic). Beskydy, 5(1): 43-54. doi: 10.11118/beskyd201205010043

LA FRANCE, M. (2002): Zu den Auswirkungen experimenteller Waldneugründungs- und Waldumbaumaßnahmen auf die saprophage Invertebratenfauna an extrem immissionsgeschädigten Kammlagenstandorten des Osterzgebirges (Sachsen). Bodenzoologisch-ökologische Untersuchungen (Oligochaeta: Enchytraeidae, Lumbricidae; Acari: Oribatida; Insecta: Collembola). Dissertation TU Dresden, Tharandt. 197 pp. + Anhang.

Lehmitz, R., RÖMBKe, J., JÄNSCH, S., KRÜCK, S., BEYLICH, A. \& GRAEFE, U. (2014): Checklist of earthworms (Oligochaeta: Lumbricidae) from Germany. Zootaxa, 3866(2): 221-245. doi: 10.11646/zootaxa.3866.2.3

MAKESCHIN, F. (1983): Bodenzoologische Ergebnisse eines Meliorationsversuches mit Weißerle auf einem ehemals streugenutzten Kiefernstandort. Mitteilungen der Deutschen Bodenkundlichen Gesellschaft, 38: 343-348.

MAKESCHIN, F. (1987): Effects of amelioration procedures on lumbricids in acidic forest soils under Pinus sylvestris. In. STRIGANOVA, B.R. (Ed.) Soil Fauna and Soil Fertility. Proceedings of the $9^{\text {th }}$ International Colloquium on Soil Zoology, Moskau, p. 392-397.

MAKESCHIN, F. \& BECK T. (1986): Langfristige Auswirkungen forstlicher Meliorationsmaßnahmen auf chemische und biologische Eigenschaften saurer Waldböden. Berichte XIII. Congress der Internationalen Bodenkundlichen Gesellschaft. Band 3: Informative Kurzfassungen, p. 849-850. 
MichaELSEN, W. (1902): Der Einfluss der Eiszeit auf die Verbreitung der Regenwürmer. Verhandlungen des Naturwissenschaftlichen Vereins in Hamburg, Dritte Folge, 9: 62-65.

MiCHAELSEN, W. (1907): Zur Kenntnis der deutschen Lumbricidenfauna. Mitteilungen des naturhistorischen Museums Hamburg, 2: 189-193.

MüLler, P. (1977): Tiergeographie. Struktur, Funktion, Geschichte und Indikatorbedeutung von Arealen. B.G. Teubner, Stuttgart, 268 pp.

PIETZSCH, K. (1962): Geologie von Sachsen. VEB Deutscher Verlag der Wissenschaften, Berlin, 870 pp.

PIŽL, V. (2001): Současný stav poznání žižal (Lumbricidae) Šumavy. In. MÁNEK, J. (Ed.) Sborník z konference Aktuality šumavského výzkumu, Srní, Czech Republic, p. 180-184.

PIŽL, V. (2002 a): Žížaly České republiky. Earthworms of the Czech Republic. Sborník Prírodovédného klubu v Uherském Hradišti, Supplementum 9: 1154.

PIŽL, V. (2002 b): Earthworms (Lumbricidae) of the Bohemian Forest. Silva Gabreta, 8: 143-148.

Schmidt, P.A., Hempel, W., Denner, M., DöRInG, N., GnÜChtel, A., Walter, B. \& Wendel, D. (2002): Potentielle Natürliche Vegetation Sachsens mit Karte 1: 200 000. Sächsisches Landesamt für Umwelt und Geologie (Ed.) Materialien zu Naturschutz und Landschaftspflege, Dresden, 230 pp.

SIMS, R.W. \& GERARD, B.M. (1985): Earthworms. Keys and notes for the identification and study of the species. E.J. Brill/ W. Backhuys, London, Leiden, Köln, København, 171 pp.

SZEDERJESI, T. (2017): The first combined checklist of earthworms of the Northeastern Mediterranean region (Clitellata: Megadrili). Opuscula Zoologica Budapest, 48(2): 77-116. doi: 10.18348/opzool.2017.2.77
ŠVARC, P. \& KULA, E. (2011): Earthworm (Lumbricidae) assemblages of forest ecosystems in the anthropogenically disturbed area of the eastern Krušne hory Mts. (Czech Republic). Journal of Forest Science, 57(6): 250-258.

VANDEL, A. (1928): La parthénogenèse géographique. Contribution à l' étude biologique et cytologique de la parthénogenèse naturelle. Bulletin biologique de la France et de la Belgique, 62: 164-281.

VANDEL, A. (1940): La parthénogenèse géographique. IV. Polyploidie et distribution géographique. Bulletin biologique de la France et de la Belgique, 74: 94-100.

Völkel, J., ZEPP, H. \& KLEBER, A. (2002): Periglaziale Deckschichten in Mittelgebirgen - ein offenes Forschungsfeld. Berichte zur deutschen Landeskunde, 76: 101-114.

WAgenBReth, O. (1978): Die Feuersteinlinie in der DDR, ihre Geschichte und Popularisierung. Schriftenreihe für geologische Wissenschaften, 9: 339368 .

WAlter, H. \& STRAKA, H. (1970): Arealkunde. Floristisch-historische Geobotanik. 2. Auflage. Ulmer, Stuttgart, $478 \mathrm{pp}$.

Wolf, L. \& AleXOWSKY W. (2008): Quartär. In. PÄLCHEN, W. \& WAlter H. (Eds.) Geologie von Sachsen. E. Schweizerbartsche Verlagsbuchhandlung, Stuttgart, $537 \mathrm{pp}$.

Wolf, L. \& SCHUBERT G. (1992): Die spättertiären bis elstereiszeitlichen Terrassen der Elbe und ihrer Nebenflüsse und die Gliederung der Elstereiszeit in Sachsen. Geoprofil, 4: 1-43.

ZICSI, A. (1965): Die Lumbriciden Oberösterreichs und Österreichs unter Zugrundelegung der Sammlung Karl Wesselys mit besonderer Berücksichtigung des Linzer Raumes. Naturkundliches Jahrbuch der Stadt Linz, 11: 125-201.

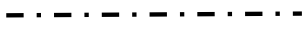

